\title{
Representing and Simulating Uncertainty of the Quality of Service of Web Services using Fuzzy Cognitive Map Approach
}

\author{
Mamoon Obiedat ${ }^{1}$ \\ Computer Information Systems \\ Al-Hashemite University \\ Zarqa, Jordan
}

\author{
Ahmad Khasawneh ${ }^{2}$ \\ Prisednt of Irbid National University \\ Irbid National University \\ Irbid, Jordan
}

\author{
Mustafa Banikhalaf $^{3}$ \\ Department of Computer Science \\ Yarmouk University \\ Irbid, Jordan
}

\author{
Ali Al-yousef ${ }^{4}$ \\ Computer Science \\ Jerash University \\ Jarash, Jordan
}

\begin{abstract}
Web services are growing rapidly to provide clients, either organizations or individuals, with multiple Internet services and to offer solutions for the integration of many applications. Quality of Service (QoS) of a Web service is the key consideration of both service providers and users. Thus, measuring the QoS requires, in addition to its normative requirements, engaging the views of clients and service providers and environmental factors. Human intervention and the environment may lead to uncertainty and result in uncertain factors in assessing QoS. In such a case, traditional computing and statistical techniques cannot provide an accurate representation of inherited uncertainties, especially when uncertain variables are connected with ambiguous (fuzzy) relationships. An alternative is to use a soft computing approach. This paper proposes a Fuzzy Cognitive Map (FCM) model as a soft computing approach that can represent and simulate the uncertainty of QoS. FCM represents the uncertain variables in the domain knowledge and their connections in the form of a signed directed graph consisting of nodes representing the variables and directed arrows representing the cause-effect relationships. In addition, it allows representing imprecise data either using numeric data, i.e. in the ranges $[0,1]$ and $[-1,1]$, or linguistic data, i.e., "low, medium, high". For calculations, FCM is converted to an adjacency matrix to find the effects of variables on each other. Scenario simulations can also be implemented to help decision makers to investigate appropriate outcomes. Finally, the proposed approach is tested by an experiment to demonstrate its reasonability and admissibility of the representation and simulation of the uncertainty of the QoS domain knowledge.
\end{abstract}

Keywords-Web services; quality of services; uncertainty; fuzzy cognitive maps; simulation; decision support systems

\section{INTRODUCTION}

A Web service is any online service that uses Web technologies and systems including standardized XML exchange systems and HTTP protocol for integrating, interacting, and exchanging data between Web applications. Nowadays, using Web services are widely used, particularly by business. Web services are an important competitive value for business. Many of the Web services do the same functions, but they may have different levels of quality [1]. Thus, Web service providers make every effort to provide high quality and performance services to satisfy their clients. Quality of service (QoS) has become an important measure to assess the degree of acceptance and appropriateness of the service provided [2]. There are different functional and non-functional QoS requirements (i.e. integrity, security, reliability, accessibility) [3]. Each of these requirement has a different role in delivering QoS and also has limitations, especially when Web Applications need to interact with each other. In addition, the nature of the Web is rapidly changing over time and numerous Web applications and technology platforms are emerging. Moreover, human and environmental factors often contribute to the selection of acceptable QoS for both its providers and users. The factors mentioned above make decision-making about QoS is dominated by the inherited uncertainty and may create several uncertain environmental and human factors/variables which in turn affect the QoS [4]. This poses a major challenge and reinforces the need for approach that can tackle this challenge and deal appropriately with such uncertain domain. Due to the inherited uncertainty, using traditional computations or even smart algorithms, such as genetic algorithms [5-7] in representing the domain knowledge are not appropriate.

Several studies have addressed QoS of the Web services under uncertainty to achieve the appropriate service required [4, 8-12]. Authors in [8] proposed a robust model that can select composite services within the uncertain QoS environment. In order to handle the uncertainty of QoS for service selection, they adopted the response time, throughput and reliability QoS attributes. On one hand, they used uncertain interval data, i.e., $[0,1]$, to express the uncertainty of the response time and service productivity. On the other hand, they used a certain dataset for service reliability. Another attempt to address QoS under uncertainty was in [9]. In this attempt, the 
researchers represented the uncertain QoS domain by a distribution function. Then, they used an approach to generate acceptable alternative services and assign uncertain scores for them. Finally, a comparison between the alternative services was performed using beneficial rules in order to report admissible services gradually. Mostafa and Zhang [10] developed two approaches; the first for single policy scenarios and the second for multiple policy scenarios. The goal of their approaches is to handle the uncertainty resulted from dynamic environments in order to efficiently compose multi-objective Web service. First, they addressed QoS criteria and challenges and conflicting objectives facing multi-objective service composition. Second, using the first approach to assign weights for the objectives of the QoS. Finally, their second approach was used in order to generate different solutions that would satisfy all the conflicting objectives of the QoS.

However, the above studies have some limitations in representing the QoS domain knowledge under uncertainty. The limitations are: the studies did not deal appropriately with uncertain data and knowledge, they did not also take into consideration the perceptions of human such as users and providers about different QoS requirements and environmental factors, and they did not differentiate between quantitative and qualitative QoS factors and relationships between these factors. Nevertheless, the uncertainty of the QoS domain is caused by several reasons, for example: dynamic environment, lack of QoS scientific information and factors, differing views between service providers and users about the factors determining the success of the service, reliability of the service as factors are affected by the current state of the Internet, and different QoS characteristics which are used by users with different levels of usage knowledge and experience [8-9].

As a result, uncertain factors/variables about QoS are most probably emerging as well as these variables may influence each other through nonlinear imprecise/fuzzy connections. Therefore, representing such uncertain domains forms a big challenge and requires a suitable approach that deals with the uncertain variables and influential connections. The goal of this paper is to propose an approach that can deal with the uncertainty of the Web service QoS as well as to represent and simulate uncertain variables and connections resulting from this inherited uncertainty. Consequently, the proposed approach could be considered as a decision support system (DSS) that would assist decision makers, i.e., service providers, in making appropriate decisions. To achieve the above goal, a soft computing approach is the best choice because it can represent and model uncertainty domains and deal with any uncertain data. In this paper, a fuzzy cognitive map (FCM) as a soft computing approach is proposed. FCM introduced by [13] is a cognitive map consisting of nodes and directed weighted edges that can represent, in a fuzzy method, uncertain variables and influential connections, respectively. FCM can be encoded into a matrix, which in turn can be simulated to provide results for analysis.

The rest of this paper is organized as follows. Section II gives an overview of FCM approach and why it has been used.
Section III presents the method proposed by this paper to represent and simulate the uncertainty of QoS. Testing the proposed method through an experimental study is presented in Section IV. Section V concludes the paper and suggests some future work.

\section{PERSPECTIVES ON FCM}

Kosko integrated fuzzy logic [14] with cognitive knowledge to produce FCM [13]. The goal of this is to address knowledge domains including inherited uncertainty. FCM approach describes the uncertain problem by collecting its uncertain variables and then assigning uncertain cause-effect relations between them. One of the advantages of FCM approach is that uncertain data can be represented in numeric interval data, such as $[0,1]$ and $[-1,1]$ or in linguistic data, such as "weak, moderate, strong". Fig. 1 shows an example of FCM. As shown from the figure, variables can influence negatively or positively on each other. For clarification, the connection value from $\mathrm{V}_{4}$ variable to $\mathrm{V}_{1}$ variable is negatively very high $(-\mathrm{VH})$, this means that if the value of $\mathrm{V}_{4}$ increases, then the value of $V_{1}$ will highly decrease, and if the value of $V_{4}$ decreases, then the value of $\mathrm{V}_{4}$ will highly increase. As shown also from the figure, the connection value from $\mathrm{V}_{6}$ variable to QoS variable is positively low $(+\mathrm{L})$, this means that if the value of $\mathrm{V}_{6}$ increases, then the value of QoS will slightly increase, and if the value of $\mathrm{V}_{6}$ decreases, then the value of QoS will slightly decrease. It is also noted from the figure that FCM approach enables loop influences. This means that the influence of a certain variable on another variable can in turn indirectly influence on that certain variable.

Another advantage of FCM approach is that it allows different relevant stakeholders (i.e., providers, clients, specialists, decision makers, etc.) regardless of their knowledge to share their views/systems by developing their own FCMs. These developed FCMs can be combined to form an expressive view/system that can comprehensively describe the knowledge domain. The individual and whole FCMs are then analyzed and simulated to produce system outcomes, which in turn reveal useful solutions, alternatives, or recommendations for decision makers in order to assist them in decision-making.

The FCM approach has proven its ability to deal with uncertainty in several domain systems $[15,16]$. Authors in [17] proposed a decision model based on big data analytics and FCM qualitative approach for improving the decision making for IT service procurement process. Authors in [18] introduced Web-mining inference mechanism using FCM inference to analyze the Web data in order to extract useful knowledge. FCM was used as a navigation to address the connections between Internet of Things and intelligent space [19]. FCM approach has also been used for modelling and simulating the uncertainty in socio-ecological, economic and environmental systems [20-23]. Next section presents the proposed FCM approach to present the uncertainty of the QoS domain and then simulate the FCM system to reach outcomes that could reveal suggestions for selecting an appropriate QoS for stakeholders. 


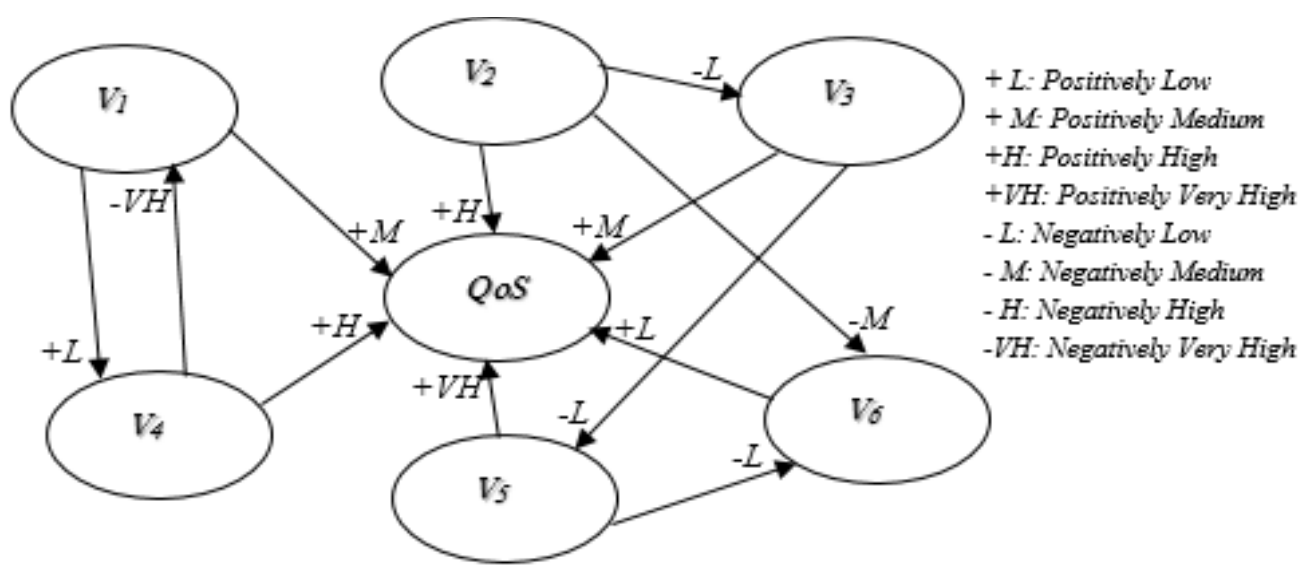

Fig. 1. Example of FCM; Connection Values in Signed Linguistic Terms.

\section{REPRESENTING AND SimULATING UNCERTAIN QoS}

Zadeh in [14] introduced soft computing approaches, such as fuzzy logic and neural networks (NN) to model systems including uncertainty in order to help in decision making. FCM combines fuzzy logic and artificial neural networks (ANN). It utilizes the fuzzy logic to represent fuzzy/uncertain values of nodes and connections. The connection values between nodes are encoded into adjacency matrix. Then, ANN is used to run the FCM system based on initial values of nodes, called initial states, until the system reaches steady values of nodes, called steady states. From the steady states, several policy scenarios can be simulated. The results of these simulations are considered as different alternative solutions offered to decision makers for selection.

\section{A. QoS Representation}

As mentioned before, any stakeholder can represent their knowledge domain in the form of FCM system. The steps of representing the uncertainty of the QoS domain knowledge using FCM approach are as follows:

a) Map nodes to represent uncertain QoS variables such as QoS requirements, factors, attributes, parameters, or other related concepts. The value of each variable takes an imprecise/fuzzy linguistic term or numeric value in the range $[0,1]$, and it is called the state of the variable.

b) Map directed arrows to represent connection weights of casual uncertain relationships between QoS variables. Each connection weight takes a fuzzy value in linguistic terms or in the range [-1, 1], see Fig. 1.

The fuzzy values (numeric in $[-1,1]$ or linguistic terms) are represented by membership functions (i.e. triangular membership functions). Each value has a degree of membership between 0 and 1 in the $[-1,1]$ universe of discourse in all membership functions. For example, Equation 1 calculates the degree of membership of any connection value represented by a triangular membership function. Fig. 2 illustrates eight linguistic terms describing the connection values (-VH, $-H,-M,-L, L, M, H$, and $V H)$ represented by eight triangular membership functions. It is worth mentioning here that the degree of membership of a linguistic term is one in the membership function that represents it and zero in other membership functions. For mathematical calculations, the degree membership values are converted to crisp values in the ranges $[0,1]$ or $[-1,1]$ using a defuzzification method, such as Centre of Gravity (COG) [24].

$\mu_{M}(x)=\left\{\begin{array}{c}\frac{x-v_{1}}{v_{2}-v_{1}}, v_{1} \leq x \leq v_{2} \\ \frac{v_{3}-x}{v_{3}-v_{2}}, v_{2} \leq x \leq v_{3} \\ 0, \text { otherwise }\end{array}\right.$

Where $v_{1} \leq v_{2} \leq v_{3}$ are the parameters of the membership function $M$ in the universe of discourse $X[-1,1], x$ is the fuzzy connection value in $X$, and $\mu_{M}(x)$ is the degree of membership of $x$ in the membership function $M$.

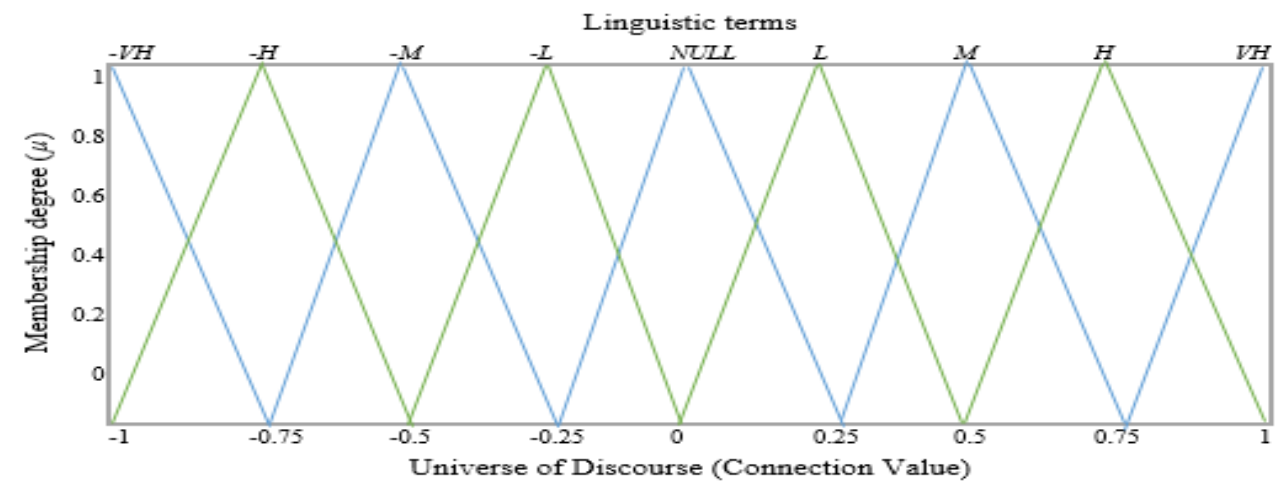

Fig. 2. Eight Linguistic Terms Represented by Eight Membership Functions in [-1, 1] Universe Of Discource. 
As stated before, any relevant stakeholder can develop their FCM system. Then, FCM systems developed by stakeholders can be combined, after converting them to adjacency matrices, using the additive method [21, 27], (Equation 2). The combined FCMs into a whole FCM can reflect a holistic view of the system [21-22, 25-26]. Then, several simulations can be conducted on any FCM system to achieve the desired outcomes. To produce a group FCM with connection values in the range $[-1,1]$, it is normalized by averaging these values.

$\operatorname{Grp}(F C M)=\sum_{k=1}^{N}\left(F C M_{k}\right)$

where $\operatorname{Grp}(F C M)$ is the resulting FCM, $N$ is the number of FCMs to be combined, and $F C M_{k}$ is the matrix of $k^{\text {th }}$ FCM.

Next section describes the simulation process to simulate the QoS domain knowledge

\section{B. Simulating $Q o S$}

Once the QoS domain knowledge is represented by an FCM system, it is easily converted to an adjacency matrix representing the QoS domain variables in its columns and rows. This adjacency matrix includes the weights of connections between variables in the range $[-1,1]$, see Table I. If the weight is greater than zero, this means that the variable in the corresponding row impacts positively by the assigned value degree on the variable in the corresponding column. The same thing is for the weight less than zero, but the impact is negatively. Finally, if the weight is zero, this means that no connection between corresponding variables.

To find the outcome of the FCM system, also called FCM inference, the following steps are performed:

a) An initial (current) state values of FCM variables in the range $[0,1]$ are represented in a vector (i.e., $V=V_{l}, V_{2}, \ldots$ $V_{N}$ ), where $N$ is the number of variables.

b) The next state value of each variable is calculated using ANN using the following Equation 3: a graphical representation of the equation is shown in Fig. 3.

$V_{i}^{t+1}=f\left(\sum_{j=1}^{N} V_{j}^{t} * w_{i j}+V_{i}^{t}\right)$

Where $V_{i}^{t+l}$ is $V_{i}$ value at time $t+1, V_{j}^{t}$ is $V_{j}$ value at time $t$, $w_{i j}$ is the connection value between $V_{i}$ and $V_{j}$, and $f$ is a threshold function, e.g., sigmoid function (Equation 4), to force the active variable to take a value in the range $[0,1]$.

$f(x)=\frac{1}{1+e^{-x}}$

TABLE I. FCM IN FIG. 1 AFTER CONVERTING TO AN ADJACENCY MATRIX

\begin{tabular}{|c|c|c|c|c|c|c|c|}
\hline & $Q o S$ & $V_{1}$ & $V_{2}$ & $V_{3}$ & $V_{4}$ & $V_{7}$ & $V_{6}$ \\
\hline$Q o S$ & 0 & 0 & 0 & 0 & 0 & 0 & 0 \\
\hline$V_{1}$ & 0.5 & 0 & 0 & 0 & 0.25 & 0 & 0 \\
\hline$V_{2}$ & 0.75 & 0 & 0 & -0.25 & 0 & 0 & -0.5 \\
\hline$V_{3}$ & 0.5 & 0 & 0 & 0 & 0 & -0.25 & 0 \\
\hline$V_{4}$ & 0.75 & -1 & 0 & 0 & 0 & 0 & 0 \\
\hline$V_{5}$ & 1 & 0 & 0 & 0 & 0 & 0 & -0.25 \\
\hline$V_{6}$ & 0.25 & 0 & 0 & 0 & 0 & 0 & 0 \\
\hline
\end{tabular}

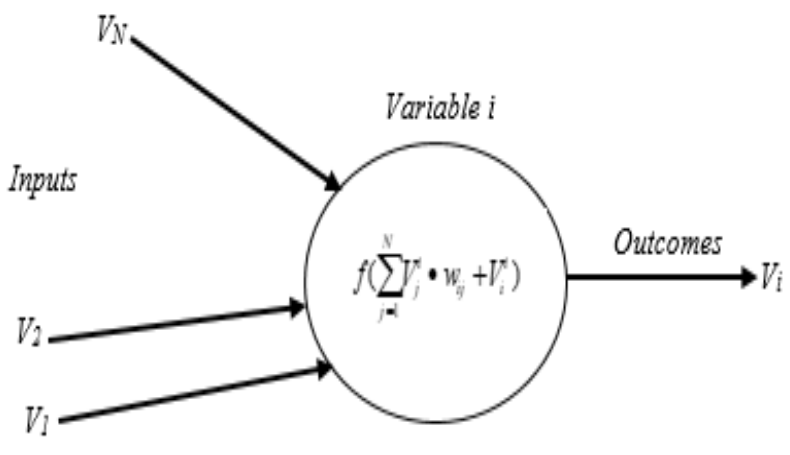

Fig. 3. A Graphical Representation of Finding a New State Value of Variable $i$ using Equation 1.

Where $x$ is the weighted total of inputs

a) The next state value of $V_{i}$ is then used as an input to find the new state values of all other variables connected to it.

b) Repeat steps b and c until the system reaches a steady state where the state values are not changed any more [27].

The resulting steady state can be considered as a system status quo, which then be used to apply different policy options. The values of states in the range $[0,1]$ reflect the amount of their influence on each other and thus on the system (i.e., 1 means high influence, 0 means no influence). Based on this, different scenarios, e.g., "what-if questions", can be performed. The results of these scenarios are then compared in order to obtain satisfy goals. For example, what will the steady state values of variables (system results) be if specified variable (s), e.g., those who have high influence, are changed in the initial state and kept fixed at a specified value while system running.

\section{EXPERIMENT STUDY}

Day after day, the use of mobile digital platforms, particularly smartphones, has increasing popularity. This is due to many mobile functions and their capabilities to run various internet applications. Therefore, a high quality of the mobile service is highly required. This paper investigates QoS of the Mobile service as an experimental study of the proposed approach. First, we selected the main factors that would influence the QoS of the Mobile service. Based on the previous studies and the views of relevant experts, these factors were "Mobile specifications (V1)", "Operating system (V2)", "Coverage (V3)", "Service cost (V4)", "Service pack (V5)", "Devices \& network technologies (V6)", "Negative user reaction (V7)", "Accessibility (V8)", and "Unlimited data plan (V9)" [28-30].

According to these variables, we then asked three of domain experts from three different businesses to represent their perceptions. They had two options; either by depicting their perceptions in the form of FCM systems or filling in an adjacency matrix with connection values that we sent them as an excel file. Fig. 4 shows an example of FCM developed by one of them. The connection weights were in the range $[-1,1]$. The three FCM systems were added to each other to form a 
group FCM system using Equation 2. We normalized the connection values of the group FCM to be within $[-1,1]$ interval, by assigning weights to the developed FCMs. These weights were equal to all FCMs, (0.33 for each FCM). The adjacency matrix of the group system is shown in Table II.

We also asked the experts to assign current values of the above factors as initial states representing the system current state. We also normalized the variable states suggested by experts using the same above process used in normalizing the connection values. Table III shows initial state values suggested by an expert, and normalized states suggested by all experts.

Then, ANN and Equation 3 were used to run the group FCM. The system reached a steady state after 29 iterations, see Table IV. Based on the system inference (steady state), it is easily to rank the variables according to the degree of their influence and hence their importance to the change in the status quo in order to reach desired outcomes.

As shown from Table IV, the state value of the variable "QoS of MS" was enhanced from 0.7 to about 0.95. This enhancement accompanied, on one side, with enchantments in the state values of the following variables: "Mobile specifications $\left(\mathrm{V}_{1}\right)$ " (from 0.47 to 0.69 ), "Operating system (V2)" (from 0.53 to 0.69), "Coverage (V3)" (from 0.67 to 0.83 ), "Service cost (V4) (from 0.7 to 0.96)", "Service pack (V5)" (from 0.37 to 0.87 ), "Accessibility (V8)" (from 0.73 to 0.9 ), and "Unlimited data plan (V9)" (from 0.43 to 0.62 ). On the other side, the state values of "Devices \& network technologies (V6)" and "Negative user reaction (V7)" variables were decreased from 0.73 to 0.66 and 0.57 to 0.09 , respectively.

The state value of $\mathrm{V}_{4}$ "Service cost" in the steady state is the highest value among all state values of the other variables. This means that it has the highest importance, and hence, it has a high impact on the QoS of the mobile service system and on the other variables as well. On the other hand, the variable $V_{7}$ "Negative user reaction" has the lowest degree of the impact on the system.

From the status quo, different policy scenario simulations can be implemented, i.e., setting a variable with a significant impact or more than one variables at a high value while the simulation process is running to observe what the system new steady state will reach [31-33].

Then, the difference between status quo and new steady states is calculated. This shows which variables, particularly "mobile service QoS", have increased and which variables have decreased. Calculated positive and negative differences mean increased and decreased variables, respectively. The amount of the calculated difference reflects the degree of increase or decrease of the variables as a result of the scenario.

Finally, making analysis and comparisons among the differences between status quo and new steady states for the different scenario simulations can reveal potential and reasonable solutions or alternatives that would enhance the "QoS of Mobile service".

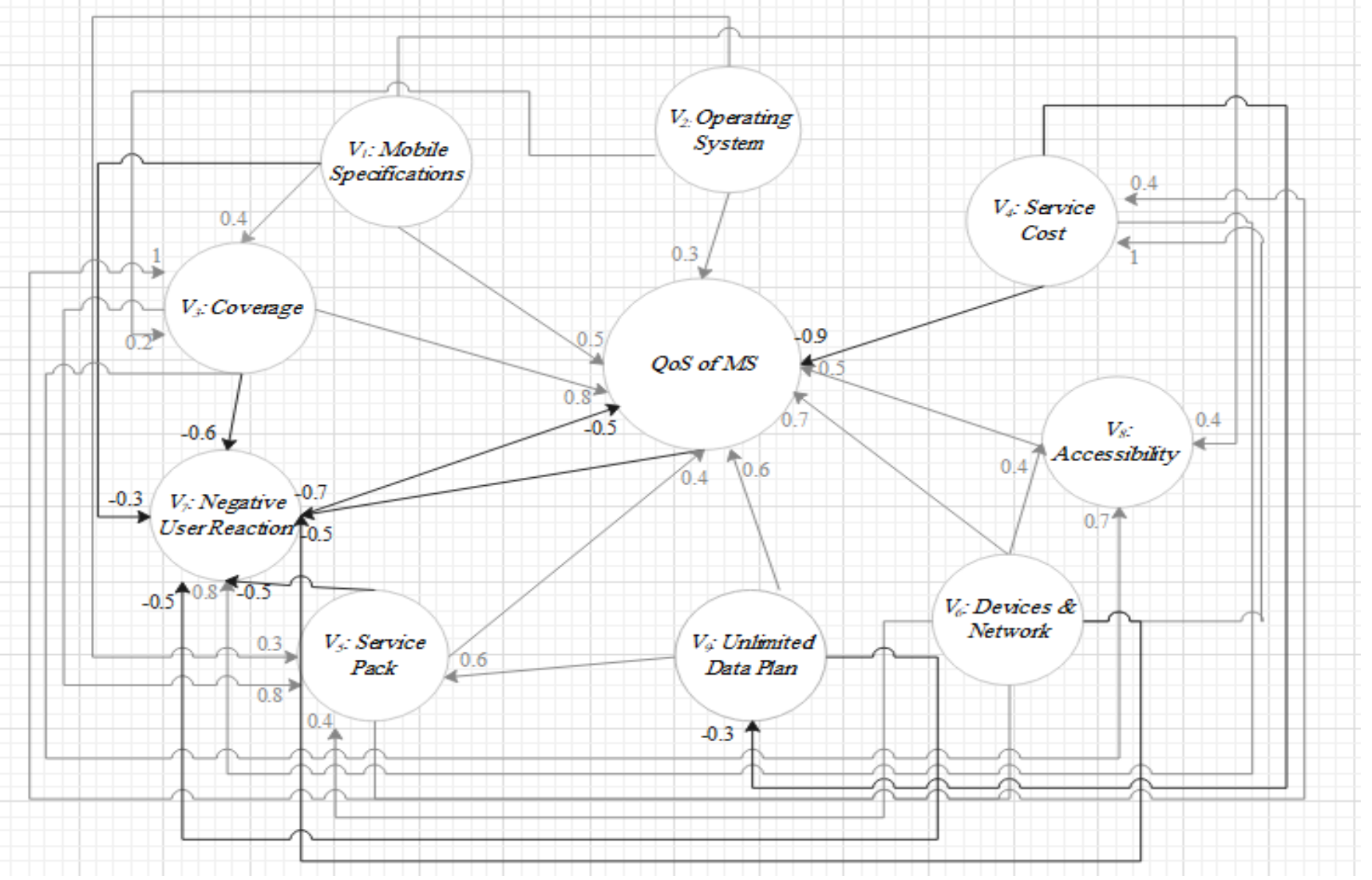

Fig. 4. FCM Developed by a Relevant Expert Representing Important Factors and their Relationships of the QoS of the MS System. 
TABLE II. FCM IN FIG. 1 AFTER CONVERTING TO AN ADJACENCY MATRIX

\begin{tabular}{|c|c|c|c|c|c|c|c|c|c|c|}
\hline & QoS of $M S$ & $V_{1}$ & $V_{2}$ & $V_{3}$ & $V_{4}$ & $V_{5}$ & $V_{6}$ & $V_{7}$ & $V_{8}$ & $V_{9}$ \\
\hline QoS of $M S$ & 0 & 0 & 0 & 0 & 0 & 0 & 0 & -0.77 & 0 & 0 \\
\hline$V_{1}$ & 0.43 & 0 & 0.07 & 0.33 & 0 & 0.13 & 0 & -0.33 & 0.4 & 0 \\
\hline$V_{2}$ & 0.23 & 0.17 & 0.1 & 0 & 0 & 0.17 & 0 & 0 & 0 & 0 \\
\hline$V_{3}$ & 0.87 & 0 & 0 & 0 & 0.83 & 0.43 & 0 & -0.73 & 0.63 & 0.07 \\
\hline$V_{4}$ & -.083 & 0 & 0 & 0 & 0 & -0.07 & 0 & 0.63 & 0 & -0.4 \\
\hline$V_{5}$ & 0.37 & 0 & 0 & 0 & 0.37 & 0 & 0 & -0.40 & 0 & 0 \\
\hline$V_{6}$ & 0.73 & 0 & 0 & 0.83 & 0.87 & 0.4 & 0 & -0.43 & 0.73 & 0.3 \\
\hline$V_{7}$ & -0.6 & 0 & 0 & 0 & 0 & 0 & 0 & 0 & & 0 \\
\hline$V_{8}$ & 0.6 & 0 & 0 & 0 & 0.37 & 0 & 0 & -0.5 & 0 & 0 \\
\hline$V_{9}$ & 0.5 & 0 & 0 & 0 & 0.33 & 0.5 & 0 & -0.47 & 0 & 0 \\
\hline
\end{tabular}

TABLE III. InItial State VAlues of ONE EXPERT AND INITIAL State VAluES OF All EXPERTS

\begin{tabular}{|l|l|l|}
\hline Variable ID & Initial states suggested by one expert & Normalized Initial states by all experts \\
\hline QoS of MS & 0.8 & 0.7 \\
\hline V1 & 0.5 & 0.466667 \\
\hline V2 & 0.8 & 0.533333 \\
\hline V3 & 0.7 & 0.666667 \\
\hline V4 & 0.8 & 0.7 \\
\hline V5 & 0.2 & 0.366667 \\
\hline V6 & 0.7 & 0.733333 \\
\hline V7 & 0.5 & 0.566667 \\
\hline V8 & 0.8 & 0.733333 \\
\hline V9 & 0.5 & 0.433333 \\
\hline
\end{tabular}

TABLE IV. THE REsults (STEADY STATES OF VARIABLES) OF THE GROUP FCM SYSTEM

\begin{tabular}{|c|c|c|c|c|}
\hline Variable ID & Initial state & Iteration 1 & \multirow{11}{*}{$\ldots .}$. & Iteration 29 steady state \\
\hline QoS of MS & 0.7 & 0.881843 & & 0.949539 \\
\hline$V_{1}$ & 0.466667 & 0.635835 & & 0.692044 \\
\hline$V_{2}$ & 0.533333 & 0.650067 & & 0.692044 \\
\hline$V_{3}$ & 0.666667 & 0.806797 & & 0.833217 \\
\hline$V_{4}$ & 0.7 & 0.919925 & & 0.956162 \\
\hline$V_{5}$ & 0.366667 & 0.78 & & 0.875281 \\
\hline$V_{6}$ & 0.733333 & 0.675536 & & 0.659046 \\
\hline$V_{7}$ & 0.566667 & 0.230705 & & 0.095841 \\
\hline$V_{8}$ & 0.733333 & 0.867074 & & 0.898568 \\
\hline$V_{9}$ & 0.433333 & 0.603483 & & 0.62124 \\
\hline
\end{tabular}

\section{ConClusion}

This paper introduces a new soft computing approach for decision makers (service providers) to support them in reaching a high level of QoS under dynamic environment dominated by uncertainty. This approach uses fuzzy cognitive map (FCM) as a model/system for representing uncertain variables existing in the QoS domain knowledge in the form of nodes. Then, each signed connection between any two variables is drawn in the form of directed arrows. The FCM is easily transformed into an adjacency matrix for mathematical calculation. This approach also allows various related stakeholders regardless their knowledge to participate in depicting their perceptions by developing their own FCMs. The developed FCM systems can be combined to obtain a comprehensive FCM system. Then, artificial neural network (ANN) is used to simulate the FCM system based on the connection values in the adjacency matrix and the initial values of variables. This simulation process ends when the system reaches a steady state where the values of the variables do not 
change. To reach an appropriate QoS, different scenarios can be simulated to see which scenario has led to a high level of QoS by comparing the outcomes of these scenarios. Finally, the proposed was experimentally examined. The results of the experiment study "QoS of Mobile service" showed the reasonableness and effectiveness of the proposed approach for representing and simulating QoS domain knowledge under inherited uncertainty.

As a future work, we intend to expand the experiment study to include more factors and connections by involving other different stakeholders such as technical supports, public users, agents, etc. We also intend to investigate the feasibility and applicability of various scenarios in order to provide useful recommendations to assist decision makers.

\section{REFERENCES}

[1] Zeng, L., et al., Quality driven web services composition. Proceedings of the 12th international conference on World Wide Web. 2003, Budapest, Hungary: Association for Computing Machinery. 411-421.

[2] Rasool, S. and U. Dulhare, A Study on Quality Aspects for Web Services. International Journal of Engineering Trends and Technology, 2016. 41: p. 232-237.

[3] Sumra, R. and D. Arulazi, Quality of Service for Web ServicesDemysti-fication, Limitations, and Best Prac-tices. 2003.

[4] Alférez, G.H. and V. Pelechano. Facing Uncertainty in Web Service Compositions. in 2013 IEEE 20th International Conference on Web Services. 2013.

[5] Zhang, C., S. Su, and J. Chen. A Novel Genetic Algorithm for QoSAware Web Services Selection. in Data Engineering Issues in ECommerce and Services. 2006. Berlin, Heidelberg: Springer Berlin Heidelberg.

[6] Zhang, C.W., S. Su, and J.L. Chen, Genetic algorithm on web services selection supporting QoS. Jisuanji Xuebao/Chinese Journal of Computers, 2006. 29: p. 1029-1037.

[7] Su, S., C. Zhang, and J. Chen. An Improved Genetic Algorithm for Web Services Selection. in Distributed Applications and Interoperable Systems. 2007. Berlin, Heidelberg: Springer Berlin Heidelberg.

[8] Chen, Y., et al., A Robust Service Selection Method Based on Uncertain QoS. Mathematical Problems in Engineering, 2016. 2016.

[9] Fu, X., et al., Discovering admissible Web services with uncertain QoS. Frontiers of Computer Science, 2015. 9(2): p. 265-279.

[10] Mostafa, A. and M. Zhang, Multi-Objective Service Composition in Uncertain Environments. IEEE Transactions on Services Computing, 2015: p. 1-1.

[11] Abdelhak, E., H. Feth-Allah, and M. Mohammed, QoS Uncertainty Handling for an Efficient Web Service Selection. 2019. 1-7.

[12] Niu, S., et al., Towards the optimality of QoS-aware web service composition with uncertainty. International Journal of Web and Grid Services, 2019. 15: p. 1.

[13] Kosko, B., Fuzzy cognitive maps. International Journal of Man-Machine Studies, 1986. 24(1): p. 65-75.

[14] Zadeh, L.A., Fuzzy logic, neural networks, and soft computing. Acm Transactions on Information Systems, 1994. 37 p. 77 - 84.
[15] Aguilar, J., A survey about Fuzzy Cognitive maps papers. International Journal of Computational Cognition, 2005. 3.

[16] Papageorgiou, E.I., Review Study on Fuzzy Cognitive Maps and Their Applications during the Last Decade, in Business Process Management: Theory and Applications, M. Glykas, Editor. 2013, Springer Berlin Heidelberg: Berlin, Heidelberg. p. 281-298.

[17] Choi, Y., H. Lee, and Z. Irani, Big data-driven fuzzy cognitive map for prioritising IT service procurement in the public sector. Annals of Operations Research, 2018. 270(1): p. 75-104.

[18] Lee, K. C., Kim, J. S., Chung, N. H., \& Kwon, S. J. Fuzzy cognitive map approach to web-mining inference amplification [Article]. Expert Systems with Applications, 2002, 22(3), 197-211.

[19] Ján Vaščák, Peter Papcun, Iveta Zolotová, Michal Puheim, "Intelligent Space Design for Robotic and IoT Applications", Cybernetics \& Informatics (K\&I), 2020, pp. 1-6.

[20] Papageorgiou, K., et al., Fuzzy Cognitive Map-Based Sustainable SocioEconomic Development Planning for Rural Communities. Sustainability, 2019. 12(1).

[21] Ozesmi, U. and S. Ozesmi, Ecological models based on people's knowledge: a multi-step fuzzy cognitive mapping approach. Ecological Modelling, 2004. 176(1-2): p. 43-64.

[22] Obiedat, M. and S. Samarasinghe, A novel semi-quantitative Fuzzy Cognitive Map model for complex systems for addressing challenging participatory real life problems. Applied Soft Computing, 2016. 48: p. 91-110.

[23] Mago, V.K., et al., Analyzing the impact of social factors on homelessness: a Fuzzy Cognitive Map approach. BMC Medical Informatics and Decision Making, 2013. 13(1): p. 94.

[24] Jang, L., Soft Computing Techniques in Knowledge-Based Intelligent Engineering Systems: Approaches and Applications. Studies in Fuzziness and Soft Computing. Vol. 10. 1997, Heidelberg: Springer.

[25] Kosko, B., Fuzzy associative memories: Neural Networks and Fuzzy Systems, in Neural Networks and Fuzzy Systems. 1992, Prentice-Hall: Englewood Cliffs, New Jersy 07632.

[26] Obiedat, M. and S. Samarasinghe, Fuzzy Representation and Aggregation of Fuzzy Cognitive Maps, in 20th International Congress on Modelling and Simulation. 2013: Adelaide, Australia.

[27] Dickerson, J.A. and B. Kosko, Virtual Worlds as Fuzzy Cognitive Maps. Presence, 1994. 3(2): p. 73-89.

[28] Husnjak, S., D. Perakovic, and I. Cvitić, Relevant Affect Factors of Smartphone Mobile Data Traffic. Promet-Traffic \& Transportation, 2016. 28: p. 435-444.

[29] Asiegbu, B., et al., Assessment of Factors Affecting Quality of Service of Cellular Mobile Network Operators in Nigeria for the Period 2010 to 2014. International Journal of Engineering and Modern Technology ISSN 2504-8848 Vol. 1 No.8 2015 www.iiardpub.org, 2015. 1: p. 27-37.

[30] Farid, F., S. Shahrestani, and C. Ruan, Quality of Service Concerns in Wireless and Cellular Networks. IBIMA Communications, 2013.

[31] Singh, K.P., et al., Evaluating the Effectiveness of Climate Change Adaptations in the World's Largest Mangrove Ecosystem. Sustainability, 2019. 11(23).

[32] Kokkinos, K., et al., Fuzzy Cognitive Map-Based Modeling of Social Acceptance to Overcome Uncertainties in Establishing Waste Biorefinery Facilities. Frontiers in Energy Research, 2018. 6(112).

[33] Mourhir, A., et al., Exploring Precision Farming Scenarios Using Fuzzy Cognitive Maps. Sustainability, 2017. 9(7). 